\title{
An Auxiliary Subxiphoid Incision in Nuss Procedure for the Treatment of Complex Pectus Excavatum
}

\author{
Xicheng Deng, Pingbo Liu, Jinghua Wang, Liwen Yi and Peng Huang \\ Department of Cardiothoracic Surgery, Hunan Children's Hospital, Hunan, China
}

\begin{abstract}
An auxiliary subxiphoid incision was utilised to facilitate pectus bar placement and minimise operative risks in complex pectus excavatum. A series of 33 patients with recurrent or severe pectus excavatum underwent Nuss procedure with this incision from March 2013 to March 2016. The median age of the cohort was 6.9 years ( 9 females vs. 24 males). The mean Haller index was 5.22. There were 12 redo cases and 18 cases with a Haller index $>6$. Four cases underwent double bar correction. There was no perioperative death or major complication. Twenty-one cases had already the bars removed when this study was initiated. During follow-up (6-30 months), three cases presented with mild depression of lateral chest wall; while in the rest, the shapes of the chest wall remained satisfactory. The application of the auxiliary subxiphoid incision in Nuss procedure can make it safer in recurrent and severe pectus excavatum with favourable postoperative cosmetic effects.
\end{abstract}

Key Words: Complex pectus excavatum, Nuss procedure, Subxiphoid incision, Recurrence.

How to cite this article: Deng X, Liu P, Wang J, Yi L, Huang P. An auxiliary subxiphoid incision in Nuss procedure for the treatment of complex pectus excavatum. J Coll Physicians Surg Pak 2020; 30(3):335-337.

Complex pectus excavatum (PE) cases, including those with severe depression of the sternum and recurrences after initial operation for pectus excavatum, pose greater operative risks and worse outcomes compared with average cases. 1,2 Though the Nuss procedure has now been standardised through more than three decades of clinical practice, the ideal procedure for complex cases remains controversial and varies from one centre to another. In this retrospective study, the authors described an additional subxiphoid incision in the Nuss procedure for the treatment of the population.

Approved by Institutional Review Board and informed consent waived, relevant data of a series of 33 cases, including 24 males and 9 females, with median age 6.9 years range (2.6 to 17 years) under operation from March 2013 to March 2016 were retrospectively collected from patients record database and during follow-up in this retrospective study. The mean Haller index was $5.22 \pm 2.37$. There were 7 cases of recurrent pectus excavatum after Ravitch procedure, 4 recurrences after Nuss procedure, and 1 after correction of pectus carinatum. Out of the series, 18 had Haller indices higher than 6 and three presented with extended depression of the anterior chest wall in association with

Correspondence to: Xicheng Deng, Department of Cardiothoracic Surgery, Hunan Children's Hospital, Changsha, 410007,

China

E-mail:justindxc@gmail.com

Received: April 05, 2019; Revised: July 15, 2019;

Accepted: July 19, 2019 a flat chest (Table I). All patients underwent subxiphoid incision assisted Nuss procedure either as a primary or redo operation. Elevation of the sternum was calculated according to pre- and postoperative computed tomography.

The patients were generally anesthetised and intubated. Prepping and draping was performed in a normal fashion. A small transverse subxiphoid incision of 2-3 cm in length at the level of the junction between the sternum and xiphoid process (Figures 1a and $1 \mathrm{~b}$ ) was firstly made and the xiphoid, as well as the retrosternal space, was bluntly mobilised using fingers or a hemostat. Subsequently, a standard Nuss procedure was performed. At the time of insertion of the introducer, a finger was put in the retrosternal space allowing for guidance of the introducer, so the introducer can be advanced retrosternally safely across the lowest point of the depression. Then the pericardium was pushed away to allow the introducer to pass through the chest wall from the planned site. The rest of the procedure was the same as a standard Nuss procedure. In asymmetrical cases, partial excision of cartilages was carried out at the discretion of individual surgeons.

All the cases had an uneventful postoperative course. There was no death or major complication including major hemorrhage, clinically significant pneumothorax, and injury to the pericardium or even to the heart. Double-bars were inserted in three relatively elder patients aged 14 to 17 years, with one or two ribs between the double bars, to achieve favourable cosmetic effects. In three cases where the pectus excavatum was asymmetrical with severe rotation of the sternum, one or 
two costal cartilages of the 4th-6th ribs were partially excised on the more severe side of the chest so as to correct stress exerted from the opposite of the sternum and achieve a roughly symmetrical cosmetic effect.

Thirteen cases had bars removed already. All the cases were followed up for 16 to 40 months. In one case with asymmetrical PE, who had one costal cartilage excised, the anterior chest wall was slightly higher on one side than on the other, yet the sternum was in normal

Table I: Characteristics of the patients.

\begin{tabular}{l|c}
\hline & Characteristics \\
\hline No. of cases (m/f) & $33(24 / 9)$ \\
\hline age (years) & Median: 6.9 (2.6 to 17) \\
\hline Case type & 21 \\
Primary cases & 7 \\
Recurrence after Ravitch procedure & 4 \\
Recurrence after Nuss procedure & 1 \\
Recurrence after correction of pectus carinatum & Mean: $5.22 \pm 2.37$ \\
\hline Haller indices & 18 cases $>6$ \\
\hline
\end{tabular}

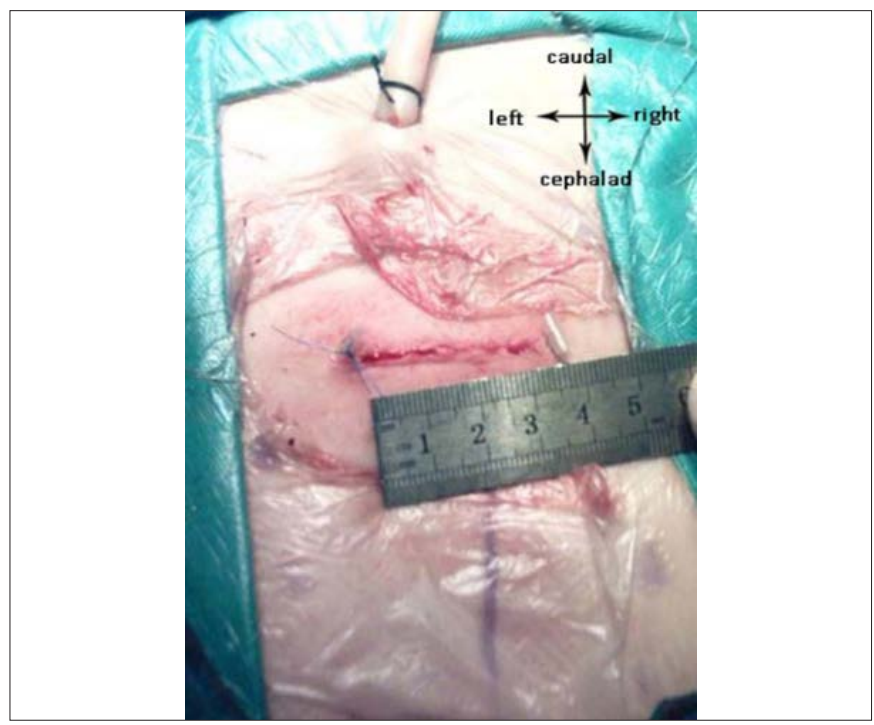

Figure 1a: Subxiphoid incison length upon the conlusion of a typical operation of the series.

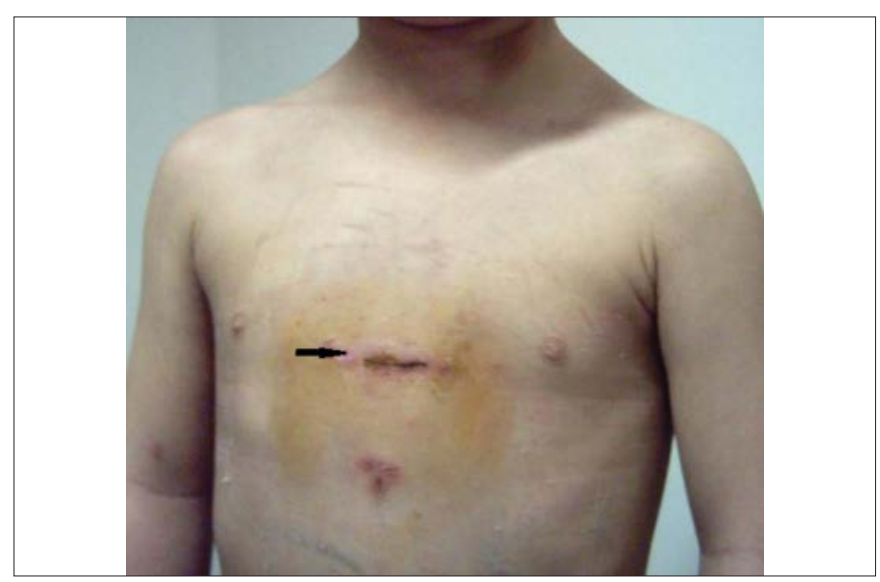

Figure 1b: The incison scar (arrow) upon discharge. position. Rough surface was found where the costal cartilage had been excised in one patient with recurrent pectus excavatum and the one after carinatum correction, whereas the elevation of the sternum was satisfactory. In one of three cases who had cartilages excised, late over-elevation of the excision side presented three years postoperatively. All the other patients had excellent postoperative chest cage contour. The sternum was elevated by $2.67 \mathrm{~cm}$ on average (range 1.98 - $3.61 \mathrm{~cm}$ ) with no significant bar displacement.

The Nuss procedure, a minimally-invasive procedure invented in 1987 by Dr. Donald Nuss, ${ }^{3}$ has now been widely used across the world for its small incision with minimal damage, technical simplicity and short recovery time. However, it comes with some major complications including major hemorrhage, pneumothorax, injury to the pericardium or even to the heart which is less common but may result in fatality. ${ }^{4}$ Currently, we use a standard thoracoscopically-assisted Nuss procedure for the majority of the patients like in most centres. However, in recurrent or high Haller index cases, cautions should be taken when performing a Nuss procedure due to adhesion in retrosternal space or the heart is so severely compressed by the wedged-down sternum that it may accidentally be perforated by a Nuss bar advancing through. Procedures for these patients vary a lot. Many advocate the use of thoracoscopy under this circumstances with standard lateral incisions, while some consider it unnecessary and even a cause of visceral injuries. ${ }^{5}$ In this series, we tried and used an auxiliary subxiphoid incision and bluntly dissected tissue and adhesion around. With this protective route and maneuver, the introducer could be safely advanced through the retrosternal space without injuring the heart. This auxiliary incision and technique was found easy, reproducible and reliable in our practice even without the use of thoracoscopy, which is consistent with published data. ${ }^{6}$ There was no death or significant intraoperative complications like heart perforation.

Based on this experience, the indications for using an auxiliary incision can be categorised and justified. In very severe pectus excavatum, heart could be deeply compressed which incur potential of injury of the heart when passing the inducer or bar through. In recurrent and secondary pectus excavatum, existing adhesion behind the sternum due to primary operations tends to be so severe that it is worth trying with a subxiphoid incision to avoid potential injury of the heart. Older patients (>14 years) with severe depression and tough ossified sternum, may require double bars, which may not proceed smoothly, and quite often with increasing possibility of injury to the heart. If double bars are needed, the authors practice is first to place a top bar to avoid blockage of the navigating finger by the bottom bar, if it was first inserted. However, in some rare situations, 
the bottom bar, has to be put in first to elevate the sternum so that a finger can reach the part where the top bar is going through. In severe asymmetrical pectus excavatum in which rotation of the sternum is necessary yet hard to be achieved, one or more costal cartilages should be considered to be excised on the more severe side through a subxiphoid incision. Then the sternum can be elevated symmetrically as much as it could be. These present mid-term results show this technique is easy to perform, reproducible and reliable. The authors recommend, considering using it in selected patients as described.

\section{CONFLICT OF INTEREST:}

Authors declared no conflict of interest.

\section{AUTHORS' CONTRIBUTION:}

XD: Performed the operations, wrote the draft, critically revised and approved the final manuscript.

PL, JW, LY, PH: Performed the operations, critically revised and approved the final manuscript.

\section{REFERENCES}

1. Guo L, Mei J, Ding F, Zhang F, Li G, Xie X, et al. Modified Nuss procedure in the treatment of recurrent pectus excavatum after open repair. Interact Cardiovasc Thorac Surg 2013. 17:258-62.

2. Redlinger RE Jr., Kelly RE Jr., Nuss D, Kuhn MA, Obermeyer RJ, Giovetsky MJ. One hundred patients with recurrent pectus excavatum repaired via the minimally invasive Nuss technique: Effective in most regardless of initial operative approach. J Pediatr Surg 2011 46:1177-81.

3. Nuss D, Kelly RE Jr., Croitoru DP, Katz ME. A 10-year review of a minimally invasive technique for the correction of pectus excavatum. J Pediatr Surg 1998. 33: 545-52.

4. Nuss D, Croitoru DP, Kell RE Jr., Govetsky MJ, Nuss KJ, Gustin TS. Review and discussion of the complications of minimally invasive pectus excavatum repair. Eur J Pediatr Surg 2002; 12:230-4.

5. Castellani C, Schalamon J, Saxena AK, Hoellwarth MG. Early complications of the Nuss procedure for pectus excavatum: A prospective study. Pediatr Surg Int 2008; 24:659-66.

6. St Peter SD, Sharp SW, Ostlie DJ, Snyder CL, Holcomb GW 3rd, Sharp RJ. Use of a subxiphoid incision for pectus bar place-ment in the repair of pectus excavatum. J Pediatr Surg 2010; 45:1361-4. 